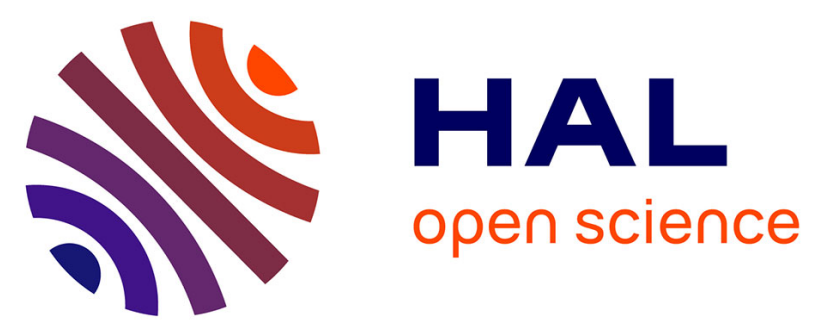

\title{
A Domain Decomposition Method for problems with structural heterogeneities on the interface: Application to a passenger ship
}

Ahmad Mobasher Amini, David Dureisseix, Patrice Cartraud, Natacha Buannic

\section{To cite this version:}

Ahmad Mobasher Amini, David Dureisseix, Patrice Cartraud, Natacha Buannic. A Domain Decomposition Method for problems with structural heterogeneities on the interface: Application to a passenger ship. Computer Methods in Applied Mechanics and Engineering, 2008, 198 (41-44), pp.3452-3463. 10.1016/j.cma.2009.06.020 . hal-00510475

\section{HAL Id: hal-00510475 \\ https://hal.science/hal-00510475}

Submitted on 4 Nov 2016

HAL is a multi-disciplinary open access archive for the deposit and dissemination of scientific research documents, whether they are published or not. The documents may come from teaching and research institutions in France or abroad, or from public or private research centers.
L'archive ouverte pluridisciplinaire HAL, est destinée au dépôt et à la diffusion de documents scientifiques de niveau recherche, publiés ou non, émanant des établissements d'enseignement et de recherche français ou étrangers, des laboratoires publics ou privés.

\section{(ㅇ)(1) $\$$}

Distributed under a Creative Commons Attribution - NonCommercial - NoDerivatives 44.0 


\title{
A Domain Decomposition Method for problems with structural heterogeneities on the interface: Application \\ to a passenger ship
}

\author{
A. Mobasher Amini*,a, David Dureisseix ${ }^{\mathrm{b}}$, P. Cartraud $^{\mathrm{a}}$, N. Buannic ${ }^{\mathrm{c}}$ \\ ${ }^{a}$ GeM, École Centrale de Nantes / CNRS UMR 6183, 1 rue de la Noë, BP 92101, F-44321 \\ Nantes CEDEX 3, FRANCE \\ ${ }^{b} L M G C$, Université Montpellier 2 / CNRS UMR 5508, CC048, Place E. Bataillon, \\ F-34095 Montpellier CEDEX 5, FRANCE \\ ${ }^{c}$ Principia Marine, 1 rue de la Noë, BP 22112, F-44321 Nantes CEDEX 3, FRANCE
}

\begin{abstract}
In this article, we extend a domain decomposition method, based on the FETIDP linear solver, to applications such as passenger ship analysis. More generally, the method is designed for large-scale elastic analysis of a structure which exhibits geometrical and structural heterogeneities, such as plate and stiffener assemblies in presence of structural details. The problem of the structural heterogeneities on the subdomain interfaces, arising from the presence of stiffeners or elastic joints on these interfaces, is addressed. A suited interface connection between subdomains modeled with plate elements in the case of a 3D assembling is proposed and tested. The selection of an efficient preconditioner is presented, and the performances and results are discussed in terms of convergence rate for several examples.
\end{abstract}

Key words: Domain Decomposition Method, FETI-DP, preconditioning, structural heterogeneity, ship structure

PACS: 45.10.Db, 46.15.Cc, 46.70.Lk

\section{Introduction}

For the design and verification of large-scale structures, such as passenger ships, there is often only one prototype produced, which is the final product. Because real-scale tests of such structures are very expensive and difficult to manage, the designers now often rely on finite element simulations.

Numerical simulation plays an increasing role in ship design, besides or associated to classification societies, responsible for approving ship design, rules and

\footnotetext{
${ }^{*}$ Corresponding author

Email addresses: ahmad.mobasher-amini@ec-nantes.fr (A. Mobasher Amini), David.Dureisseix@lmgc.univ-montp2.fr (David Dureisseix), patrice.cartraud@ec-nantes.fr (P. Cartraud)

Preprint submitted to Computer Methods in Applied Mechanics and EngineeringDecember 24, 2008
} 
regulations. These rules and regulations are based on the experience feedback obtained by monitoring a large number of vessels; they often lead to oversizing design practice. Extensive use of numerical simulations started only recently in this field, partly due to the advent of new designs and innovative structures, for which there are no feedback yet, and consequently, for which rules and regulations are not reliable. In addition, increasing the worldwide competition in structural optimization and safety level requires an accurate evaluation of the safety margins and more detailed numerical analyses.

Obtaining local results at the scale of structural details is a challenge because the large ratio of the details characteristic length to the whole structure length leads to a huge finite element model. Due to the memory and processor limits, solving this kind of problem with a classical direct technique is prohibitive, especially when this problem is embedded into an optimization loop. Using a simplified global model of the structure is therefore mandatory.

A classical approach (the so-called global-local method) can be decomposed in three steps. In the first step, a local or microscopic problem is solved on the fine scale model of the detail to derive its global or macroscopic (homogenized) behavior, that is used in the global model of the structure, see Figure 1. In a second step, the global model is solved to obtain overall results on the structure, and finally a post-processing step allows to recover the solution at the small scale of the details. A similar technique is used in the hierarchical Dirichlet projection method $[1,2]$. Using these strategies encounters several limits. First, the definition of the homogenization process is not obvious, since the structural details are not periodic. Therefore, assumptions have to be made on boundary conditions for the local problems (fine-scale) dealing with structural details. Second, the results of the global problem with a coarse mesh (coarse-scale) are used as boundary conditions for the post-processing phase to recover the smallscale solution. In this way, artificial edge effects may appear that pollute the local stress computations. In other word, with this approach, the finite element models for both scales (fine and coarse) are independent of each others, and the global computation is uncoupled from the local one.

This coupling can be improved using iterative techniques between local to global problems, see $[3,4,5]$. However, these methods may be not effective for the analysis of a large-scale problem.

To overcome the underlying difficulties, several features are required: (i) an accurate incorporation of the details into the global model, (ii) a fast and efficient global computational procedure, and (iii) a good accuracy on local stresses. These goals can be achieved with domain decomposition methods (DDM). Actually, the first step in the solution process is to get a fine-scale mesh of the various details, from which the entire global model of the ship is built on (global fine mesh). Concerning the global computation step, the degrees of freedom of the details are condensed to reduce the size of the problem. Finally, the last step is concerned with recovering the local solution on each detail (or subdomain). If the condensation is performed on suited coarse degrees of freedom, it leads to a numerically homogenized behavior; if the recovering of the local solution is consistent with this homogenization, it can be interpreted 


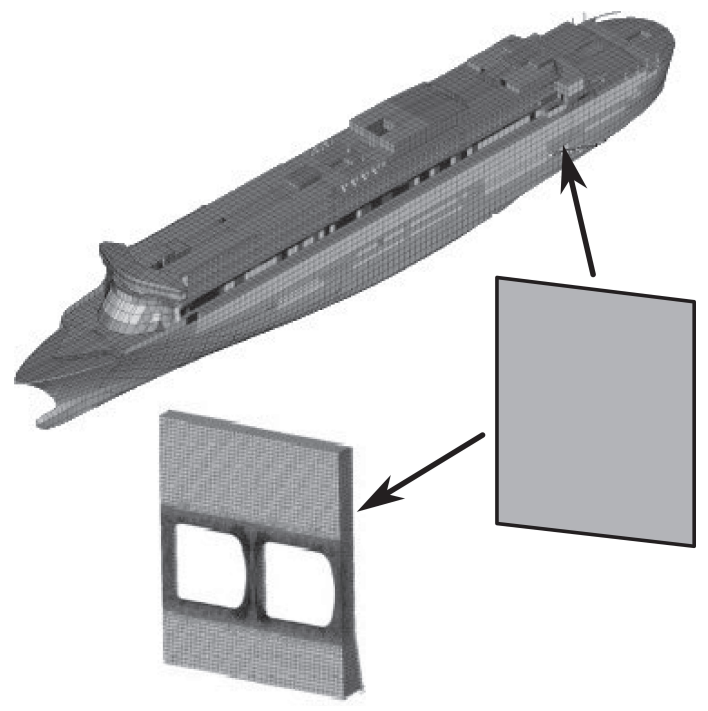

Figure 1: Finite element model at coarse-scale: left and middle, and fine scale: right.

at the relocalization phase of the homogenization procedure.

Moreover, domain decomposition methods are both an efficient and flexible tool in structural analysis for the large systems of equations arising from the discretization of elasticity problems $[6,7,8]$. As iterative approaches, they often outperform direct methods when the size of the model increases, and they allow a parallel treatment of the resolution phase. In this article, we are not concerned with the parallelization of the resolution, but we focus on the modularity, especially for adapting this method to structures with geometrical heterogeneities on the interface.

Here, we consider the FETI-DP algorithm $[9,10]$ which belongs to the family of the FETI (Finite Element Tearing and Interconnecting) methods [11]. In this method the interface constraints are enforced by using a Lagrange multiplier, while some other continuity constraints on primal displacement variable ('corner' degrees of freedom of the interfaces) are prescribed at each iteration. These last constraints provide a coarse problem and should be selected so that the iterative method converges rapidly.

When using the FETI-DP method for the analysis of passenger ship structures, several difficulties arise. In such structures, the presence of different structural elements, like plates for panels and beams for stiffeners, leads to strong heterogeneities on the interfaces of subdomains. As a consequence, it is difficult to obtain a large convergence rate for the iterative solution method. The choice of a suited preconditioner is an important issue, and in addition, for the $3 \mathrm{D}$ plate assembling, a special treatment of the interface gluing condition is required.

The objective of this article is to develop an efficient FETI-DP approach for 
heterogeneous structures with the application to passenger ship analysis. For this purpose, the remainder of this article is organized as follows. In Section 2 , the finite element modeling of the passenger ship structure is presented. In Section 3 the choice of the FETI-DP domain decomposition method is justified, and the basics of this method are recalled. Section 4 introduces the issues and difficulties to apply this approach to the case of ship structural analysis, and more generally to complex stiffened structures made as assemblies of several structural elements such as plates and beams. It also describes the dedicated solution method of the interface problem. Section 5 presents the numerical results. Finally, Section 6 concludes the article and suggests several future works.

\section{Passenger ship structure modeling}

A passenger ship is a large and complex structure due to the numerous design details (porthole, cabin doors ... ) which are much smaller in size than the global length of the ship. Another feature lies in the presence of a network of additional stiffeners, with different sizes, on the whole structure. In practice, this structure is modeled by an assembling of different structural elements, like panels and stiffeners. The presence of the details and the association of these elements lead to several geometrical and mechanical heterogeneities.

The stiffeners are usually classified into two categories: primary and secondary stiffeners, see Figure 2.

The primary stiffeners are explicitly modeled, due to their large participation to the overall stiffness of the structure. They are discretized with beam elements according to Bernoulli theory, and their size is identical to the plate element one, see Figure 3.

In the design office, due to different specifications, several discretized models (at different scales and for different design stages) are used, for instance:

- For the global behavior of the structure leading to the global architecture of the design, and to satisfy the regulation requirements on the basis of numerical tests, the so-called coarse finite element model is used. This model derives from the ship architecture whose main structural elements are explicitly modeled: hull, decks, bulkheads and primary stiffeners. By contrast, structural details — windows, doors, secondary stiffeners - are smoothed through the homogenized behavior of coarse elements, computed from the fine mesh shown in Figure 3, right. Typically, an entire panel delimited by the nodes of the network of primary stiffeners is modelled with a single finite element (Figures 1 and 3 );

- For the local stresses verification, and to take into account the details (windows, doors ...) a model at a finer scale is required;

- For assessment of local fatigue in very localized areas (such as window corners) a much refined model is required, usually with 3D elements. 

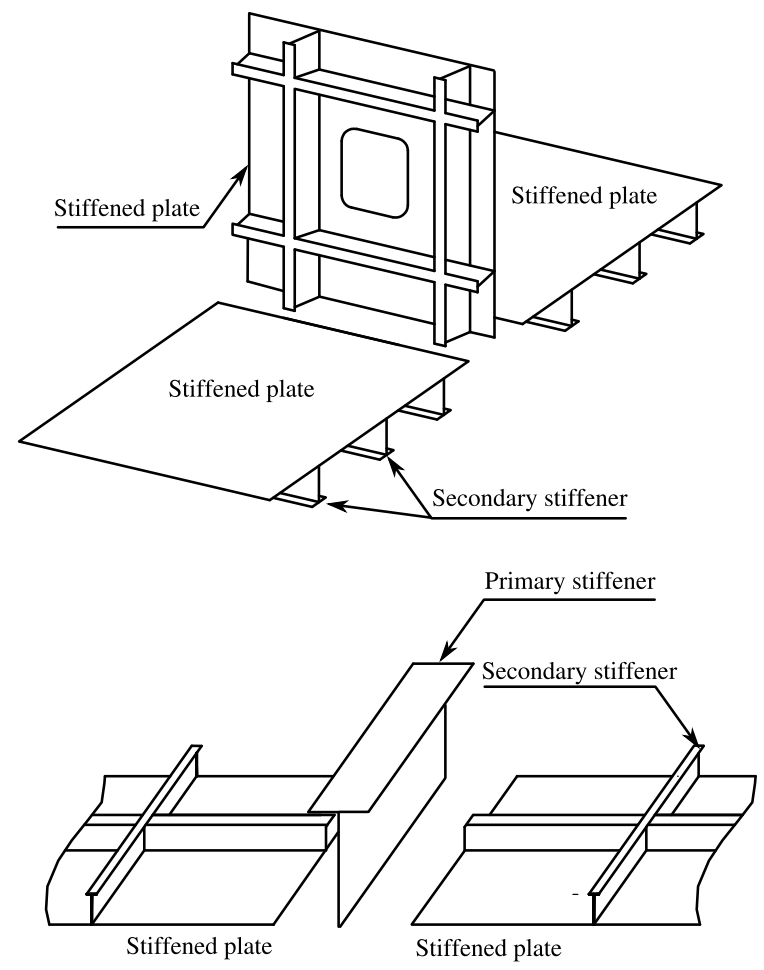

Figure 2: Primary and secondary stiffeners
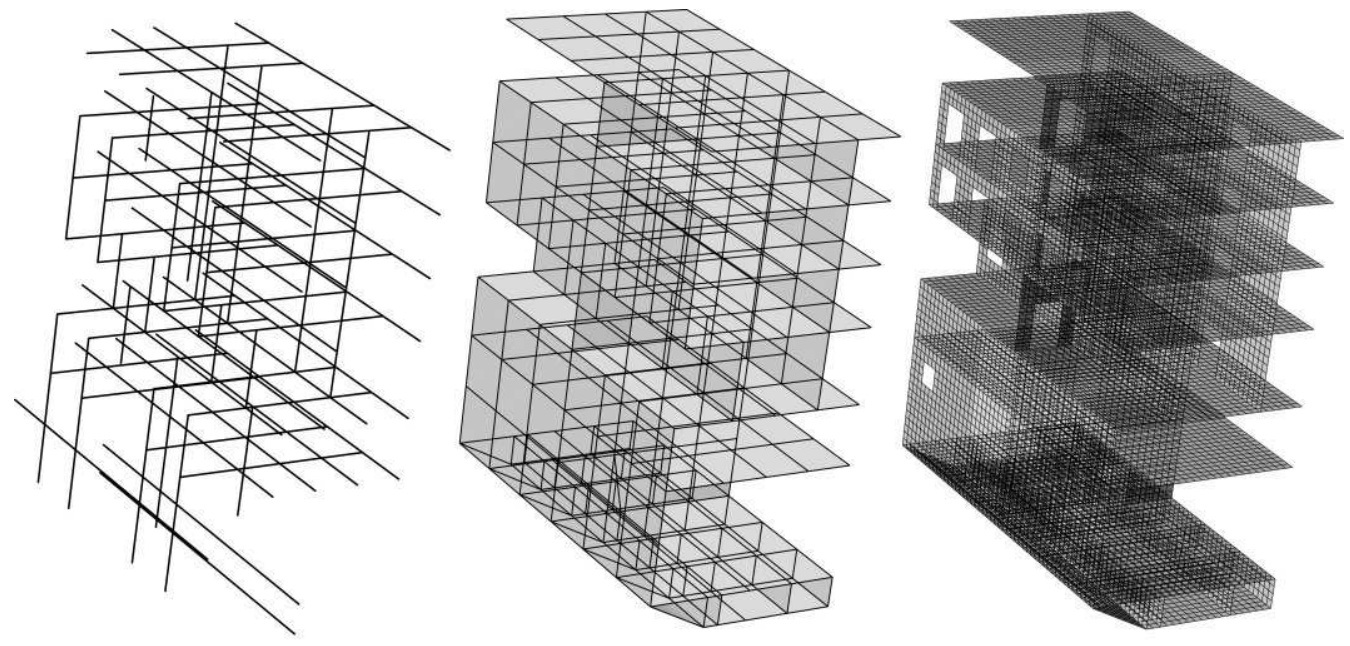

Figure 3: A typical half slice of a passenger ship; left: coarse model of primary stiffeners, middle: coarse model of panels, right: fine complete model 
The ship hull is a curved surface, but due to the small curvature with respect to the size of elements that will be used, and because shell finite elements are more cumbersome to manage, a discretization with plate finite elements is classically used. It leads to a small faceting of the surface as an additional approximation, and to a small overestimation of its stiffness. In this article, we will use DKT and DKQ (Discrete Kirchhoff Triangle and Discrete Kirchhoff Quadrilateral) finite elements [12].

In this work, we are mainly interested with the interactions between the coarse model and the scale needed for local stresses verification. Nevertheless, the proposed approach could be used to embed more than two scales and/or models together. Our aim is to use a coarse finite element model which has the same topology as the one used in the design office. However, this model has to be strongly coupled with the underlying fine mesh model, which is performed in this work through the use of a domain decomposition method described in the next section.

\section{A domain decomposition method suited to a passenger ship struc- ture}

Usually with a multilevel domain decomposition method, the reference (and finely discretized) problem is split into subdomains, with a more or less automatic process using algorithms and softwares that are often based on graph theory, see $[13,14]$ for instance. In the context of a passenger ship design, as mentioned previously, the structure presents a natural sub-structured architecture, which relies on intersections between the hull, bulkheads, decks and primary stiffeners. The classical or industrial method of analysis (global-local method) in design office is based on this sub-structuring to construct the coarse finite element model. With this natural decomposition in subdomains, it appears that a domain decomposition method is well adapted to the ship structural analysis. Moreover, with their multilevel version, this kind of method allows to restore the coupling between the different scales along iterations.

\subsection{Choice of a domain decomposition method}

In ship structure analysis, the coarse problem is already provided, since we choose to identify it with the coarse scale finite element model, see Figure 3, comprising both the coarse discretization of the panels and of the primary stiffeners. Therefore, each coarse finite element has its corresponding fine scale finite element model, with its structural details (window, door, secondary stiffener ....). This last one is then identified as a subdomain. The coarse discretization of the primary stiffeners is also derived in a consistent manner from a fine mesh, but we choose to consider herein all the fine scale primary stiffeners in a unique special subdomain. The corresponding subdomain possesses a large number of degrees of freedom but has a loose connectivity, and therefore is not a critical bottleneck for computation point of view, Figure 4.

As a summary, the coarse discretization of the panels (with macro plate super-elements) and of the primary stiffeners (with macro beam super-elements) 

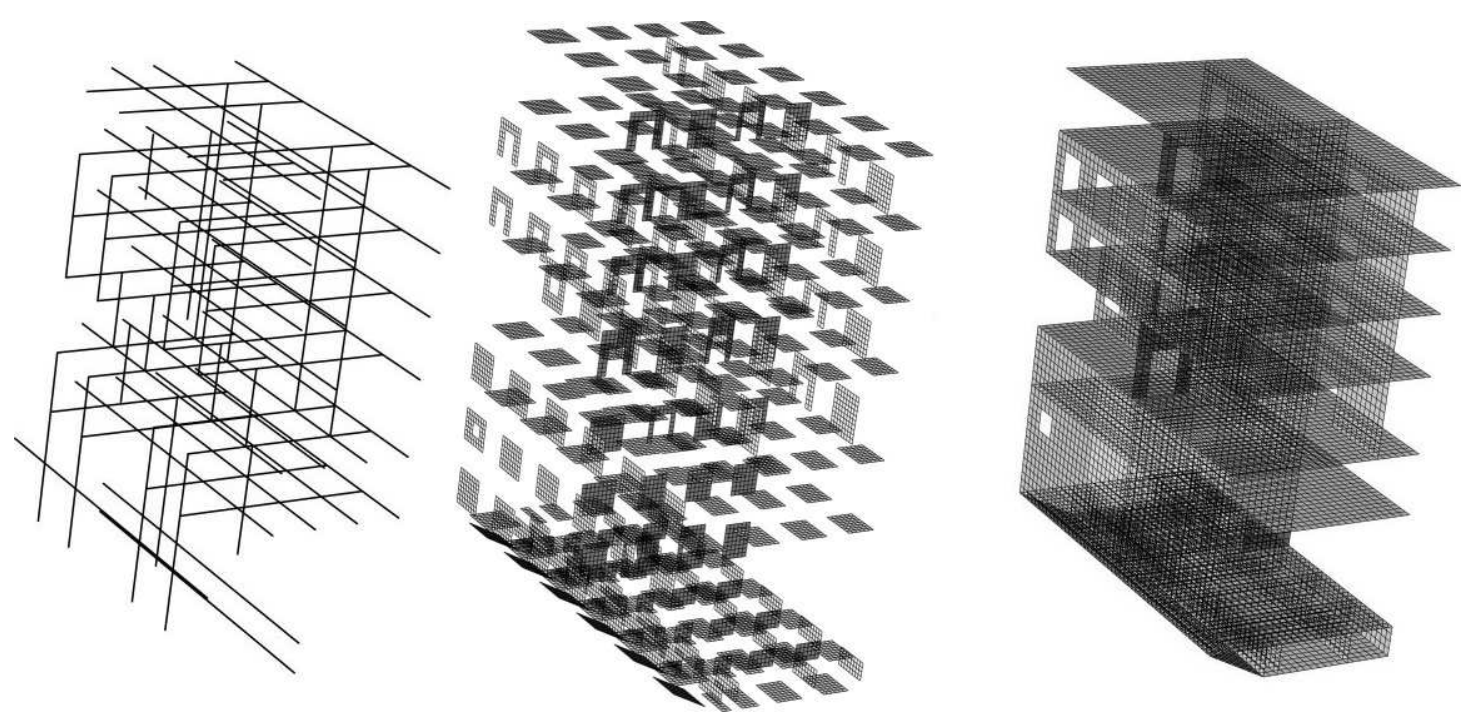

Figure 4: A typical half slice of a passenger ship (fine discretization); left: subdomain constituted of primary stiffeners, middle: classical subdomains, right: fine complete model

constitutes the problem at the the macro level. It is also the coarse problem of the multilevel DDM (with 'corner' degrees of freedom). The subdomains constitute the substructured problem at micro level; they are either:

- The fine discretization of a panel together with its associated structural details;

- The special subdomain with the fine discretization of all the primary stiffeners.

The next step consists in choosing a suited domain decomposition method to a passenger ship simulation problem. Numerous domain decomposition methods have been proposed in the literature [15]. To reach numerical scalability, a domain decomposition method needs for a multilevel feature, mainly to be able to build a coarse problem that maintains the convergence rate weakly dependent on the number of substructures. Most recent domain decomposition methods suited to structural problems are now equipped with such a coarse problem; for instance, one may refer to FETI-DP [10, 16], CBDD [17], smoothed aggregation techniques [18, 19], LATIN micro-Macro [20, 21]. Moreover, when this coarse problem is related to an homogenized model of the same structure, this provides an efficient tool to deal with heterogeneous structures.

Among all these domain decomposition methods, we choose the FETI-DP method for the following reasons:

- The subdomains are connected together in a strong manner at the corner node of the interface and in the a weak manner at other interface nodes. The corner nodes that are common between the subdomains are called 
coarse nodes. In this way we obtain a coarse mesh corresponding exactly to the industrial global mesh. Moreover, as detailed in the following, the coarse finite element model constitutes a structural homogenization of the detailed subdomains, and the compatibility of coarse displacement field is ensured automatically with neighboring coarse elements.

- From a numerical point of view, the FETI-DP method is the most improved version of the FETI family of methods. It is therefore robust and computationally efficient, see [9, 10]. It's coarse problem size is smaller than the FETI method. This comes from the fact that in the FETI-DP method, there are no floating subdomain, because of the corner nodes common to several subdomains. In addition, at each iteration of the iterative solver, the FETI method must solve twice the problem coarse, instead of once in the FETI-DP method.

\subsection{Basics of the FETI-DP method}

To keep this paper as self-contained as possible, the classical FETI-DP method $[9,10]$ is herein briefly recalled by considering the following global symmetric positive semi-definite static problem:

$$
K u=f
$$

where $K$ is stiffness matrix and $f$ is an arbitrary load vector.

Let us consider the domain $\Omega$, for which the discretization of the elastic structural model leads to the problem (1). By splitting the domain into $N_{s}$ non-overlapping subdomains with matching interfaces, $\Gamma^{(s, q)}=\partial \Omega^{s} \cap \partial \Omega^{q}$, one obtains the global interface as follows:

$$
\Gamma=\bigcup_{s=1, q>s}^{s=N_{s}} \Gamma^{(s, q)}
$$

In this case, $K^{s}, u^{s}$ and $f^{s}$ denote the the stiffness matrix, displacement vector degrees of freedom and load vector associated to the subdomain $\Omega^{s}$, respectively.

One interesting point of the domain decomposition methods, beside their simple mechanical interpretation, is that they can easily be explained from a purely algebraic point of view, i.e. directly from the matrix form of the problem.

In each subdomain, the unknowns are partitioned into global corner degrees of freedom denoted by subscript ' $c$ ', and remaining degrees of freedom denoted by subscript ' $r$ '. The $K^{s}, u^{s}$ and $f^{s}$ can be partitioned as follows:

$$
K^{s}=\left[\begin{array}{ll}
K_{r r}^{s} & K_{r c}^{s} \\
K_{r c}^{s^{T}} & K_{c c}^{s}
\end{array}\right], u^{s}=\left[\begin{array}{l}
u_{r}^{s} \\
u_{c}^{s}
\end{array}\right], f^{s}=\left[\begin{array}{c}
f_{r}^{s} \\
f_{c}^{s}
\end{array}\right]
$$

The remainder degrees of freedom, $r$, can be partitioned into interior degrees of freedom, $i$, and boundary degrees of freedom, $b$, see Figure 5 . Therefore, the block vector, $u_{r}^{s}$ and $f_{r}^{s}$ can be written as follows:

$$
u_{r}^{s}=\left[\begin{array}{l}
u_{i}^{s} \\
u_{b}^{s}
\end{array}\right], \quad f_{r}^{s}=\left[\begin{array}{l}
f_{i}^{s} \\
f_{b}^{s}
\end{array}\right]
$$




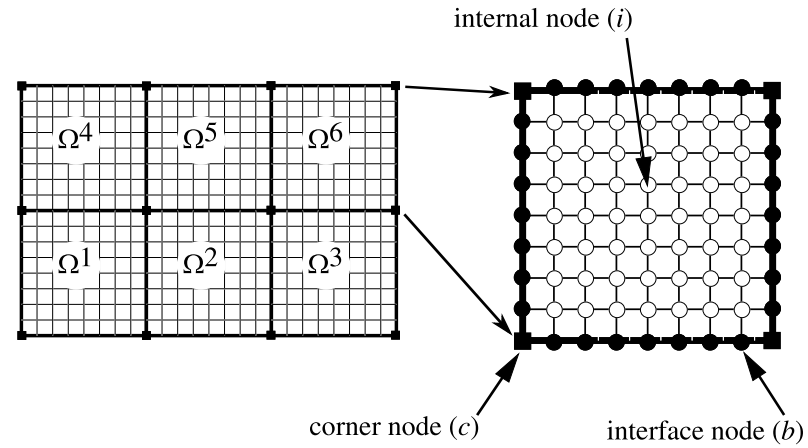

Figure 5: Classification of the subdomain nodes

One can consider $u_{c}$ as the global vector of corner degrees of freedom and $u_{c}^{s}$ as its restriction to subdomain $\Omega^{s}$. The Boolean and localization matrices, $B_{r}^{s}$ and $L_{c}^{s}$, are introduced to locate degrees of freedom as follows:

$$
\begin{aligned}
& B_{r}^{s} u_{r}^{s}= \pm u_{b}^{s} \\
& L_{c}^{s} u_{c}=u_{c}^{s}
\end{aligned}
$$

In references $[9,10]$, it was shown that solving problem (1) is equivalent to solving the following subdomain based problem:

$$
\begin{gathered}
K_{r r}^{s} u_{r}^{s}+K_{r c}^{s} L_{c}^{s} u_{c}+B_{r}^{s T} \lambda=f_{r}^{s} \quad \text { for } s=1, \ldots N_{s} \\
\sum_{s=1}^{N_{s}} L_{c}^{s T} K_{c r}^{s} u_{r}^{s}+\sum_{s=1}^{N_{s}} L_{c}^{s T} K_{c c}^{s} L_{c}^{s} u_{c}=\sum_{s=1}^{N_{s}} L_{c}^{s T} f_{c}^{s} \\
\sum_{s=1}^{N_{s}} B_{r}^{s} u_{r}^{s}=0
\end{gathered}
$$

where $\lambda$ is a vector of Lagrange multipliers introduced on $\Gamma$ to enforced the continuity equation (8) of the displacement vector $u$. To guaranty the nonsingularity of the matrix $K_{r r}^{s}$, each subdomain should contain at least three non colinear corner nodes, [22].

Using the equations (6), (7) and (8) and after some algebraic transformations, one obtain the following dual-primal problem (using Lagrange multiplier, $\lambda$, and primal displacement, $u_{c}$, as unknowns):

$$
\left[\begin{array}{cc}
F_{I_{r r}} & F_{I_{r c}} \\
F_{I_{r c}}^{T} & -K_{c c}^{\star}
\end{array}\right]\left[\begin{array}{c}
\lambda \\
u_{c}
\end{array}\right]=\left[\begin{array}{c}
d_{r} \\
-f_{c}^{\star}
\end{array}\right]
$$


where

$$
\begin{aligned}
& F_{I_{r r}}=\sum_{s=1}^{N_{s}} B_{r}^{s} K_{r r}^{s-1} B_{r}^{s T} \\
& F_{I_{r c}}=\sum_{s=1}^{N_{s}} B_{r}^{s} K_{r r}^{s-1} K_{r c}^{s} L_{c}^{s} \\
& d_{r}=\sum_{s=1}^{N_{s}} B_{r}^{s} K_{r r}^{s-1} f_{r}^{s} \\
& f_{c}^{\star}=\sum_{s=1}^{N_{s}} L_{c}^{s T}\left(f_{c}^{s}-K_{c r}^{s} K_{r r}^{s-1} f_{r}^{s}\right) \\
& K_{c c}^{\star}=\sum_{s=1}^{N_{s}} L_{c}^{s T}\left(K_{c c}^{s}-K_{c r}^{s} K_{r r}^{s-1} K_{r c}^{s}\right) L_{c}^{s}
\end{aligned}
$$

The matrix $K_{c c}^{*}$ is sparse; its pattern is that of a stiffness matrix obtained by considering only the coarse elements on the whole structure, as super-elements.

By condensing $u_{c}$ on $\lambda$ in equation (9), we obtain the following symmetric positive definite dual interface problem:

$$
F_{I} \lambda=D_{r}
$$

with:

$$
\begin{aligned}
& F_{I}=F_{I_{r r}}+F_{I_{r c}} K_{c c}^{\star}{ }^{-1} F_{I_{r c}}^{T} \\
& D_{r}=d_{r}-F_{I_{r c}} K_{c c}^{\star}{ }^{-1} f_{c}^{\star}
\end{aligned}
$$

Usually, the different domain decomposition methods use an iterative algorithm to solve the interface problem (11) to avoid the explicit assembly of the left-hand-side. Concerning FETI-DP, the iterative algorithm is a conjugate gradient. This iterative algorithm does not require to assemble explicitly the interface operator (12), but to compute a matrix-vector product $F_{I} \lambda$ at each iteration.

From an implementation point of view, the time consuming operations in the FETI-DP approach [10] are the following ones. First, during initialization:

- The initial factorization of the stiffness matrix of each subdomain $K_{r r}^{s}$;

- The initial local resolutions on each subdomain (as many as local coarse degrees of freedom) to build explicitly the basis $V_{r c}^{s}=K_{r r}^{s^{-1}} K_{r c}^{s}$;

- The initial assembly and factorization of the coarse matrix $K_{c c}^{\star}=\sum_{s} L_{c}^{s T}\left(K_{c c}^{s}-\right.$ $\left.K_{c r}^{s} V_{r c}^{s}\right) L_{c}^{s}$

and second, during each iteration, the matrix-vector product $F_{I} \lambda$ which is performed with the following steps:

- With a given multiplier $\lambda$, for each subdomain: disassemble it into $\lambda^{s}=$ $B_{r}^{s T} \lambda$, compute $f_{c}^{s}=V_{r c}^{s} \lambda^{s}$ and solve $K_{r r}^{s} u_{r}^{s}=\lambda^{s}$; 
- Assemble $f_{c}=\sum_{s} L_{c}^{s T} f_{c}^{s}$, then solve $K_{c c}^{\star} u_{c}=f_{c}$;

- For each subdomain: disassemble it into $u_{c}^{s}=L_{c}^{s} u_{c}$, then compute $v_{r}^{s}=$ $V_{r c}^{s} u_{c}^{s}$

- Finally, assemble the result into $F_{I} \lambda=\sum_{s} B_{r}^{s}\left(u_{r}^{s}+v_{r}^{s}\right)$.

The success of these methods also lies in the use of a preconditioner suited to the problem to be solved. It will be discussed in details in Section 4.2.

\section{Specific issues for the simulation of passenger ships}

The classical FETI-DP method has been presented in the previous section. Its application to the case of a passenger ship raises mainly two problems which are discussed in this section. The first one derives from the ship topology, which leads to interface with can interconnect more than two subdomains. The second problem comes from the structure heterogeneity, which originates ill-conditioned interface problem.

\subsection{Interface between subdomains}

In the case of a passenger ship, some portions of the interface may connect more than two subdomains, see Figure 3. For matching meshes, exact compatibility is ensured over $\Gamma$ simply by collocation the nodes as usual. For instance, using the subscript $b$ for the interface nodes, we can write the following relations in case of three subdomains on the interface, see Figure 6.

$$
\begin{aligned}
& u_{b}^{(1)}=u_{b}^{(2)} \\
& u_{b}^{(2)}=u_{b}^{(3)} \\
& u_{b}^{(3)}=u_{b}^{(1)}
\end{aligned}
$$

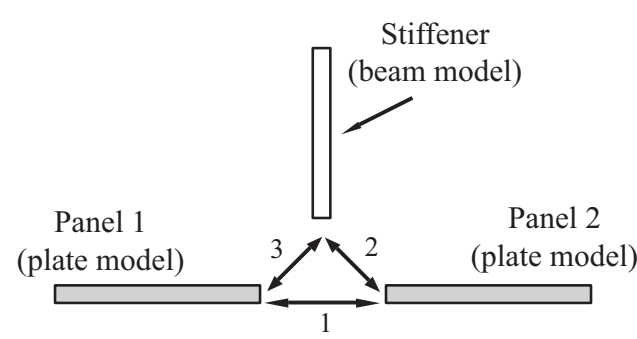

Figure 6: Multiplicity on the interface

It is obvious that, one constraint is redundant and may be omitted. We will see later in this work that, for numerical efficiency, the complete set of constraints has to be considered in practice. A similar situation occurs when the interface is the cross point of different structural parts, such as the hull and 
the desk. The first tests on complex assemblings for ship analysis were presented in [23].

Additionally, in passenger ships design, particular technological components such as joints can be used in the assembling of sub-structures. They usually possess a low stiffness to accommodate for manufacturing discrepancies in dimensions of these substructures. Such joints are usually flat enough to be discretized with 'joint' finite elements (eventually several elements in the thickness direction for multi-layered joints) with the particularity of having null geometrical thickness, but a particular constitutive relation. These components participate to heterogeneities on interfaces between subdomains, but with a different nature from the stiffeners. They nevertheless could be managed within the same domain decomposition framework, as described in Appendix B.

\subsection{Structural heterogeneity and preconditioning}

For the interface problem (11), the preconditioner must be a good approximation of the inverse of $F_{I}$. Since $F_{I}$ is not explicitly assembled, the preconditioner must be independent of the value of the element of the matrix $F_{I}$.

In this article, we do not develop a new preconditioner for FETI-DP method, but we use the weighted Dirichlet and Lumped preconditioners that are presented in [6] as follows:

- Dirichlet preconditioner:

$$
\bar{F}_{I_{r r}}^{D^{-1}}=\sum_{s=1}^{N_{s}} W^{s} B_{r}^{s}\left[\begin{array}{cc}
0 & 0 \\
0 & S_{b b}^{s}
\end{array}\right] B_{r}^{s T} W^{s}
$$

where $S_{b b}^{s}$ is the local Schur complement defined by:

$$
S_{b b}^{s}=K_{b b}^{s}-K_{i b}^{s^{T}} K_{i i}^{s^{-1}} K_{i b}^{s}
$$

- Lumped preconditioner:

$$
\bar{F}_{I_{r r}}^{L^{-1}}=\sum_{s=1}^{N_{s}} W^{s} B_{r}^{s}\left[\begin{array}{cc}
0 & 0 \\
0 & K_{b b}^{s}
\end{array}\right] B_{r}^{s^{T}} W^{s}
$$

$W^{s}$ is a subdomain-based diagonal weighting matrix that may accounts for subdomain heterogeneities [24].

The Dirichlet preconditioner is more computationally expensive than the lumped preconditioner, because it involves numeric factorization of $K_{i i}^{s}$ and

back substitutions solving $K_{i i}^{s^{-1}} \beta$ for each subdomain during each iteration of the preconditioner conjugate gradient algorithm. However, in general, it reduces the number of required iterations and improves the overall computational efficiency for the fourth-order plate and shell problems, and the lumped preconditioner is mostly used for second-order problems [9]. One can give a mechanical interpretation of the FETI-DP method and Dirichlet preconditioner as follows. 
At each iteration $k$ a new Lagrange multiplier field $\lambda^{k}$ is computed. Then, for each subdomain $\Omega^{s}$, a load vector is imposed on its interface boundary and a subdomain displacement fields, $u_{s}$, is obtained. Except at convergence, the subdomain solution $u_{r}^{s}$ is not continuous throughout the subdomain interfaces, and a displacement jump is evaluated as:

$$
r^{k}=\sum_{s=1}^{N_{s}} B_{r}^{s} u_{r}^{s k}
$$

The preconditioning of the residual, $r^{k}$, by the Dirichlet operator corresponds to a inverse sequence of local manipulations: The jump of displacement field is evaluated on the interfaces and splitted between adjacent subdomains; a Dirichlet problem is solved in each subdomain to evaluate the traction field corresponding to such jumps. The interpretation of the lumped preconditioner is similar, except that it assumes that all the stiffness of a subdomain is lumped at its interface boundary. Equally splitting the residual between the connected subdomains leads to a weighting matrix, $W$, whose entries are the inverse of the multiplicity of each multiplier $\lambda$ dof; the multiplicity is the number of subdomains connected by the corresponding multiplier. This method is appropriate in the cases where interfaces between the subdomains are homogeneous. For the case where the subdomains are heterogeneous on the interface this choice is not mechanically sound and leads to a lack of optimality for the preconditioners. Rixen and Farhat [24] proposed to use the so-called smoothed preconditioner, that embeds multiplicity-based scaling factors.

The diagonal weighting matrix entries are computed with the diagonal coefficients of the stiffness matrices of the connected subdomains [24]. For the subdomain $s$ to which the multiplier $j$ connects the subdomain $t$ :

$$
W_{j}^{s}=\frac{K_{j j}^{t}}{\sum_{r \in \delta(j)} K_{j j}^{r}} \quad \text { with } j \in \Gamma^{(s, t)}
$$

assuming that $j$ represents the same degree of freedom shared by the $r$ subdomains and where $\delta(j)$ is the set of subdomain connected on the interface.

It should be noted that, this result is mechanically consistent. The stiffer subdomains capture less than the displacement jump in compare to the flexible subdomains. Moreover, it can be seen that in homogeneous case $\left(K_{j j}^{(s)}=K_{j j}^{(r)}\right)$, the weighting matrix is found to be $\operatorname{diag}(1 /$ multiplicity).

For more informations the reader can refer to $[11,24,10,16]$.

\section{Numerical examples}

Before applying the FETI-DP method to the analysis of a ship structural model, the approach is tested on simple examples.

To assess the algorithm convergence on the interface problem (11), at each iteration $k$, an error $e^{k}$ is computed:

$$
e^{k}=\frac{\left\|r^{k}\right\|_{2}}{\left\|D_{r}\right\|_{2}}
$$


with:

$$
r^{k}=F_{I} \lambda^{k}-D_{r}
$$

where \|\|$_{2}$ is the $L^{2}$-norm of a vector, and $r^{k}$ is the residual of the interface equation at iteration $k$. The stopping criterion is $e^{k} \leqslant \epsilon$, where $\epsilon$ is set to $10^{-6}$ to assess the convergence.

To assess the convergence properties, in the remainder of this article, the convergence curves depict the error (in energy norm) with respect to a reference discretized solution computed with a direct solver.

Pre and post-processing are performed with the finite element code Cast3M (CEA Saclay, France) and all computations are carried out by developing a finite element code in Matlab ${ }^{\mathrm{TM}}$.

\subsection{Membrane behavior of stiffened plates}

The objective of this example is to study the effect of the redundancy on the Lagrange multipliers when the multiplicity is greater than 2 .

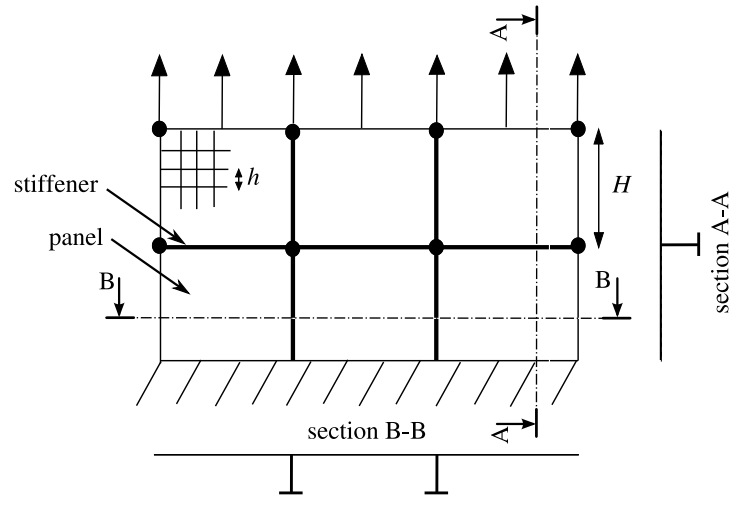

Figure 7: Stiffened plate

One considers herein the 2D static response in plane stress analysis of a plate with stiffeners, see Figure 7. It is related to a rectangular domain, clamped on the bottom edge and submitted to a uniform traction force on the top.

The classical triangular and rectangular plane stress finite element (with two degrees of freedom at each node) are used to model the panels, and twonode Bernoulli beam elements (with three degrees of freedom at each node: two displacements and one rotation) to model the stiffeners.

The isotropic elastic linear material parameters are: Young's modulus $E=$ $200 \mathrm{GPa}$, and Poisson's ratio $\nu=0.3$ for plate and beam elements. In addition, the beam element characteristics are: cross section area $S=0.00295 \mathrm{~m}^{2}$ and bending moment of inertia $I=1.447110^{-6} \mathrm{~m}^{4}$.

In this example, the stiffeners lie on the interfaces of the decomposition into subdomains. It is recalled that one subdomain is composed with the stiffeners. There are therefore three subdomains on each interface (two panels and one 
stiffener). Two computations are performed on the stiffened plate. In the first one, two Lagrange multipliers are considered (two equations in (13) are taken into account), while three are involved in the second one, see section 4.1. In addition, in order to highlight the influence of structural heterogeneity on the method efficiency, another computation is done for the same plate but without stiffeners. In both cases, the Dirichlet preconditioner is used, which can be either multiplicity-based or scaling-based (see (18)), these two approaches being equivalent in the case of the plate without stiffeners.

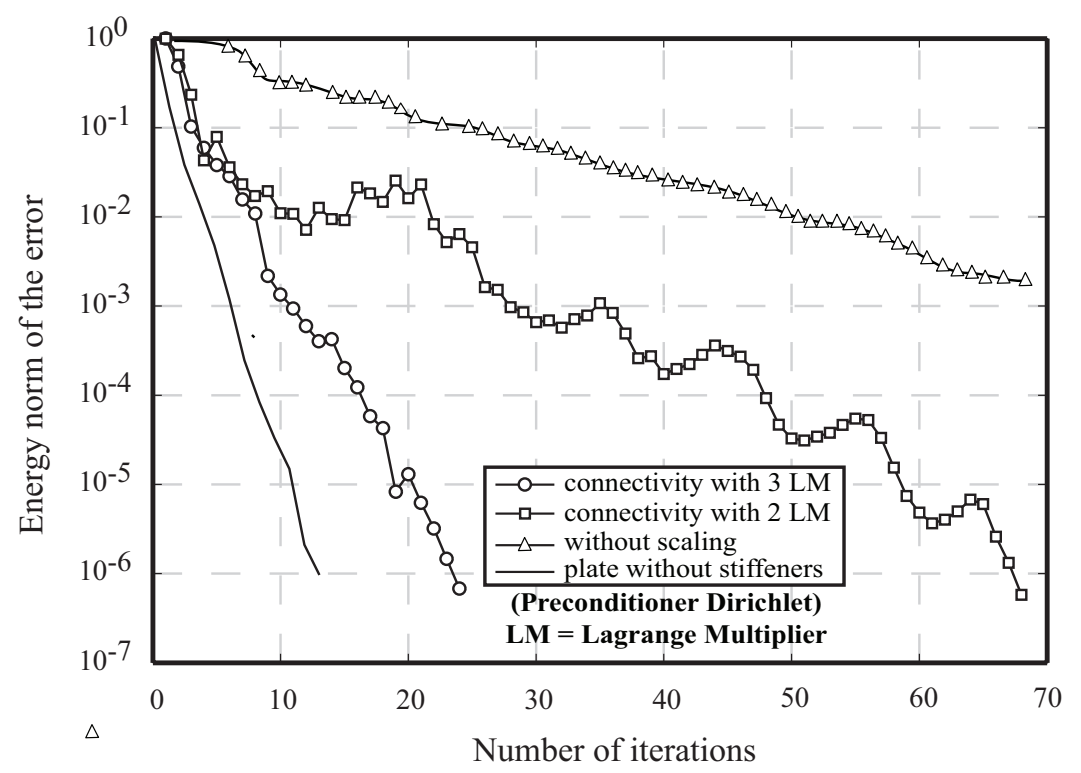

Figure 8: Influence of the connectivity on the convergence rate

The numerical results in term of convergence rate are reported in Figure 8. For the stiffened plate, it can be seen that considering three Lagrange multipliers instead of two allows to improve the convergence rate. Moreover, it can be seen that the use of a scaling-based Dirichlet preconditioner is more efficient than a multiplicity-based one. However, when two Lagrange multipliers are considered, the convergence remains slow and appears to be not monotonous. By contrast, with three Lagrange multipliers, the convergence rate is significantly improved and has the same order of magnitude as the plate without stiffeners (for this latter problem the size of the problem is lower than for the stiffened plate).

\subsection{Junction between plates on the subdomain interfaces}

In a passenger ship model, there are numerous junctions between plate finite element. It is therefore important to check the performance of the FETI-DP method for such kind of structures. 
When dealing with the case of an assembling of non-coplanar plates, see Figure 9, by the previous DDM, we observe that the convergence rate is not monotone, see Figure 10 (curves number 1 and 2).

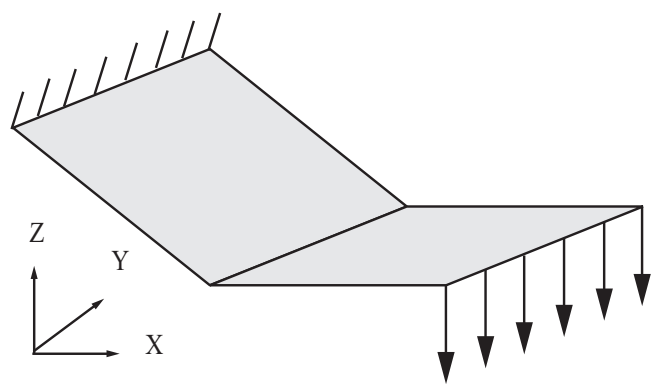

Figure 9: Folded plate (non coplanar junction)

As it will be explained in the following, this poor convergence rate is related to the choice of connection conditions between the subdomains.

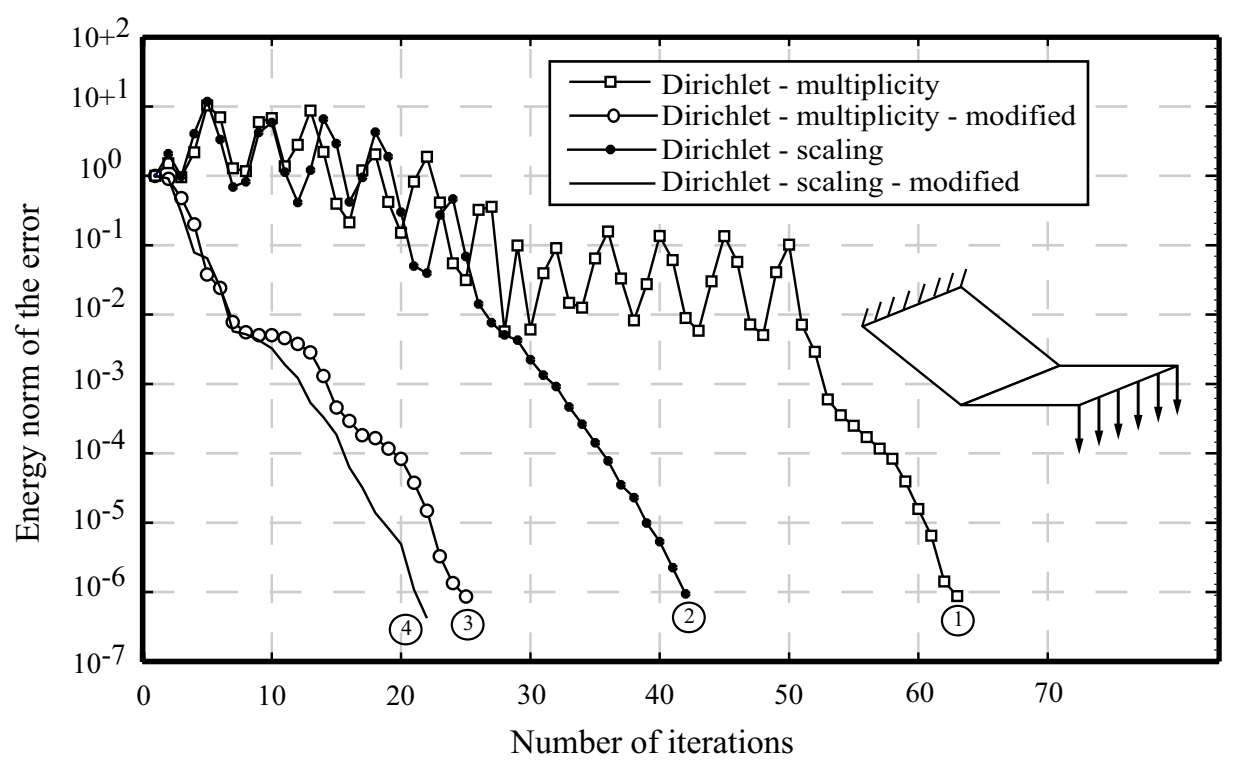

Figure 10: Convergence rates, folded plate case

For these two computations, a perfect connection on the interface is prescribed with the continuity constraint of all the global degrees of freedom on the two sides of the interface.

With the plate finite element of Kirchhoff theory (DKT and DKQ) these global degrees of freedom are the 3D nodal displacements and rotations, and they are not independent from each other. Indeed, the formulation of the plate 
is defined in the plane coordinate of the plate. It involves five degrees of freedom (three scalar displacements and two rotations) since the rotation along the normal to the plate is not defined. Moreover, according to the kinematics of the plate based on Kirchhoff theory, the normal displacement and the in-plane rotations are linked together [12].

These dependencies are the source of the weak convergence when the interface is the junction line between a 3D assembling of plates. The condition number of the problem is then artificially high, and the numerical behavior is not optimal. We therefore propose to use a modified connection condition, by following the developments in $[25,26,27]$. The main idea is to enforce the minimal number of degrees of freedom continuity constraints that lead to the discrete continuity of the finite element kinematic fields.

If we consider two subdomains $\left(\Omega^{1}\right.$ and $\left.\Omega^{2}\right)$ connected on the interface $\Gamma$, meshed with discrete Kirchhoff elements, we enforce the continuity of the all displacement degrees of freedom ( $u, v, w$ in the global basis) but only the continuity of the rotation in the direction $\underline{t}$ given by the interface line $\Gamma$ :

$$
\begin{gathered}
{\left[\begin{array}{c}
u_{1} \\
v_{1} \\
w_{1}
\end{array}\right]=\left[\begin{array}{c}
u_{2} \\
v_{2} \\
w_{2}
\end{array}\right]} \\
\underline{t} \cdot \underline{\theta}_{1}=\underline{t} \cdot \underline{\theta}_{2}
\end{gathered}
$$

The justification of this choice is detailed in Appendix A. It is easily implemented with the modification of the localization matrices $L_{r}^{(s)}$, that will now contain the components of the direction vector of the interface, $\underline{t}$, for rotational degrees of freedom. It must be noted that such an adaptation had already been done for the problems of contact, where the normal vector of the contact is used [28]. For additional details on such plate junctions, the reader may refer to $[29,30,31,32,33]$ and references herein.

Using this continuity condition successfully significantly improve the convergence rate, see Figure 10, curves 3 and 4 . For this homogeneous case, few differences appear when comparing the use of the simple multiplicity weighting, or the scaling version. Finally, note that this modified continuity condition does not change the discrete converged solution.

\subsection{Application to a multiple plate assembly}

To check the proposed technique on a more complex assembling, with both in-plane and out-of-plane junctions, and when several connections happen on the same interface, the problem depicted on Figure 11 is considered.

The structure is subjected to an overall bending and traction loading, with a linear distribution of forces applied on one end, the other end being clamped. The material is considered as an isotropic one with the following parameters: Young's modulus $E=200 \mathrm{GPa}$ and Poisson's ratio $\nu=0.3$. The following data are used for the geometry: length $L=3.0 \mathrm{~m}$, breadth $B=2.0 \mathrm{~m}$, height $H=2.0 \mathrm{~m}$, with a uniform thickness $t=0.005 \mathrm{~m}$ for the plates. For this study, 


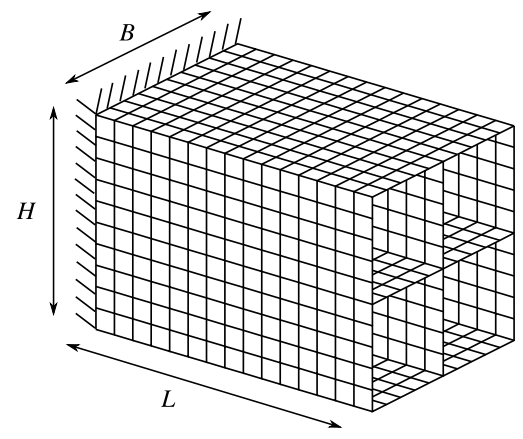

Figure 11: Multiple plate assembly

the structure was divided into 36 subdomains. The reference numerical solution is still obtained with the finite element code Cast3M. The convergence rate of the FETI-DP method for two cases (classical interface connection and modified interface connection) is presented in Figure 12. This test confirms the previous results.

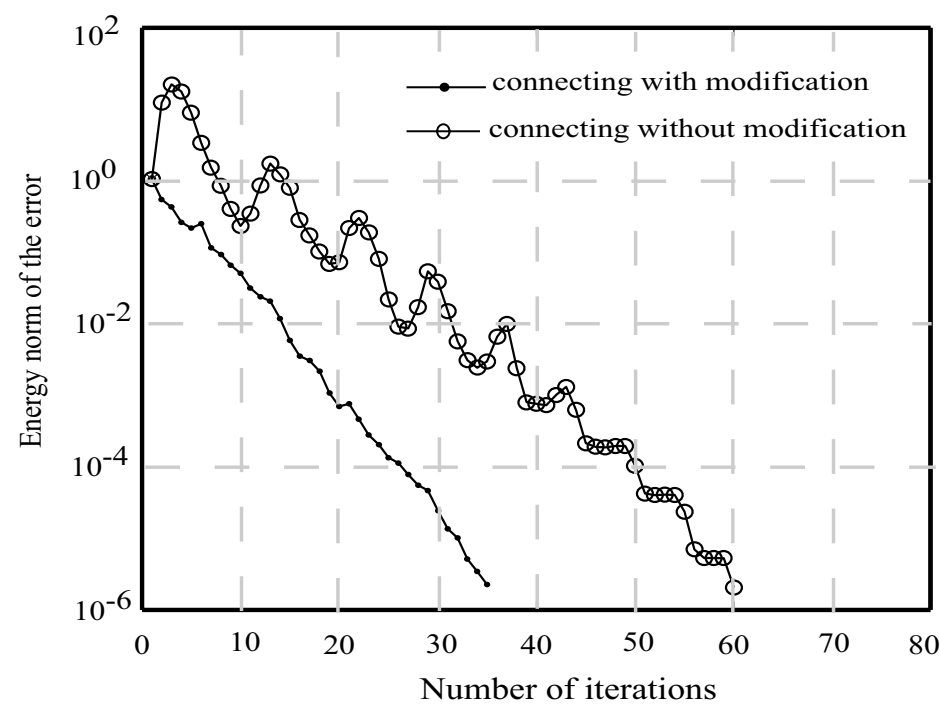

Figure 13: Convergence rate in energy norm

\section{Conclusions}

In this article, the FETI-DP method has been adapted to large-scale structural analysis, in presence of structural and geometrical heterogeneities on the interface of the subdomains. This method is well suited for the incorporation of structural details at the micro scale (the fine mesh of the subdomains). 


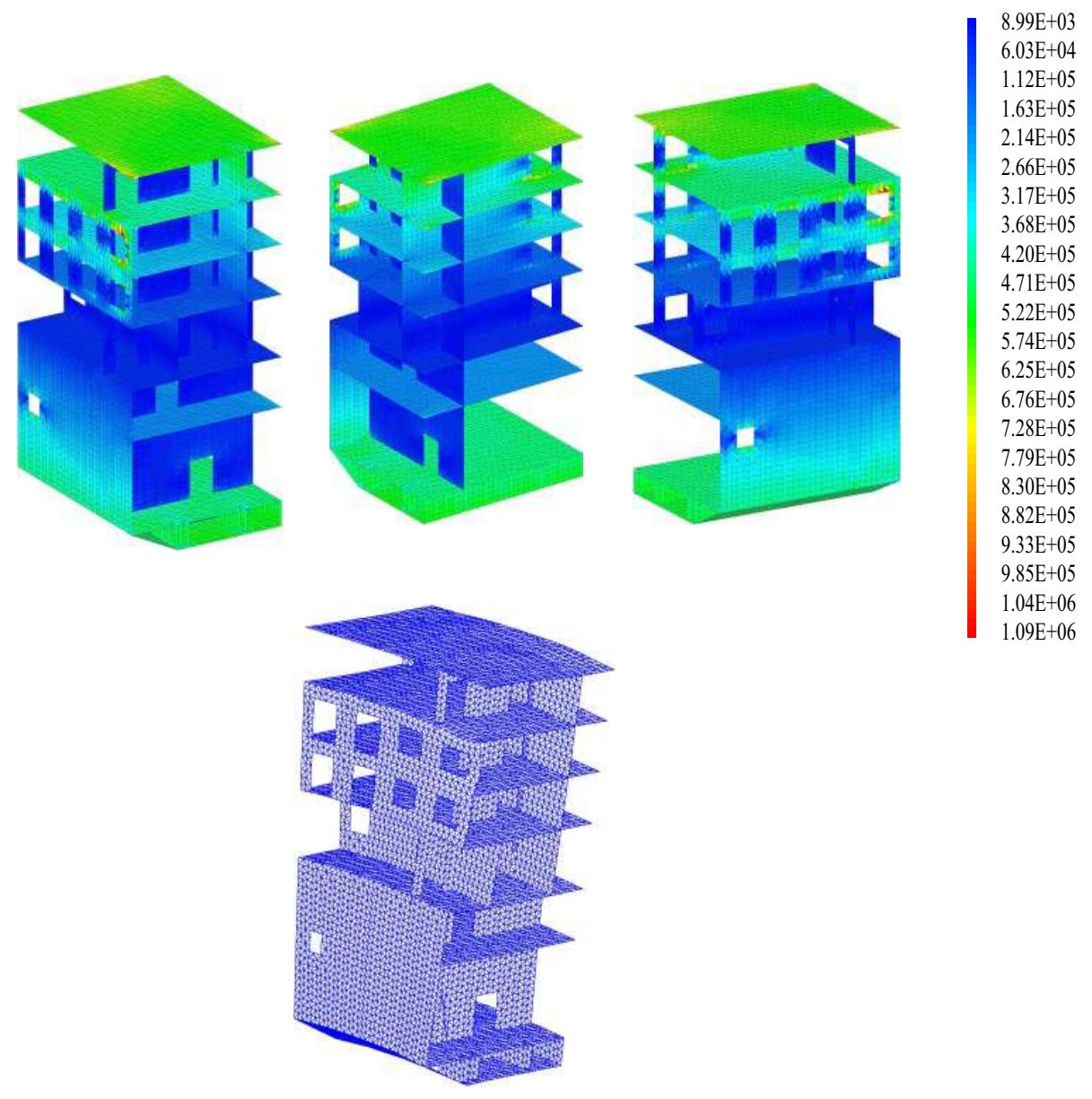

Figure 14: Von Mises stress distribution and deformed shape 
This method has been illustrated on the example of a passenger ship structure. Within this context, the proposed approach reuses the classical coarse model used in design offices as the so-called coarse space of the multilevel domain decomposition method, and build automatically its numerically homogenized behavior.

However, the performance of any type of domain decomposition method is linked to that of the underlying iterative solver. For a resolution with a conjugate gradient, it is useful to develop an efficient preconditioner. In the presence of structural heterogeneities, the use of a rescaling approach is mandatory to preserve the optimality of the domain decomposition. We also highlighted the difficulties inherent to the $3 \mathrm{D}$ connection of the plates on the interface that require a specific gluing condition. Moreover, we have shown that when an interface interconnects more than two subdomains, the best performance is obtained when all the gluing conditions are considered.

With the simultaneous use of these tools, we were able to exemplify the effectiveness of the method. A typical validation example was performed, on a portion of a passenger ship. A two-scale analysis is therefore performed on a representative ship structure. The coarse (homogenized) model is provided independently of the fine mesh and is not derived by an automatic domain decomposition. Moreover, the particular structure of the ship is taken into account with a special treatment of the primary stiffeners. The developed method has been validated by comparison of a reference solution, obtained from the direct finite element solution of the fine model of the whole structure.

This method can be improved by reducing the computation time. This can be achieved by restricting the use of a fine mesh for the subdomains in the zones of interest, while the others are replaced by homogenized coarse elements, see [34]. Future works will also concern an even more localized and refined analysis (3D analysis of very small details where fatigue leads to a critical area for crack initiation) with a 3-scale model. A 2-scale vibration analysis is also of interest to assess customer comfort requirements.

\section{A. Junctions between plate subdomains}

When we use a finite element based on Kirchhoff theory (DKT and DKQ elements), special attention should be paid to the connection between the subdomains. Let us consider two plate elements $\Omega_{e}^{r}$ and $\Omega_{e}^{s}$, belonging to the two subdomains $\Omega^{r}$ and $\Omega^{s}$, connected along a common interface element $\Gamma_{e}$. We consider a local coordinate basis in each subdomain, on the common interface, $\left(\underline{t}_{r}, \underline{n}_{r}, \underline{z}_{r}\right)$ and $\left(\underline{t}_{s}, \underline{n}_{s}, \underline{z}_{s}\right)$, see Figure 15 .

For standard plate models, the perfect connection of the continuum displacement and rotation fields is:

$$
\begin{array}{rrr}
\forall p \in \Gamma_{e}, & \underline{u}^{s}(p)=\underline{u}^{r}(p) & \text { for the displacement } \\
\underline{\theta}^{s}(p)=\underline{\theta}^{r}(p) & \text { for the rotation }
\end{array}
$$




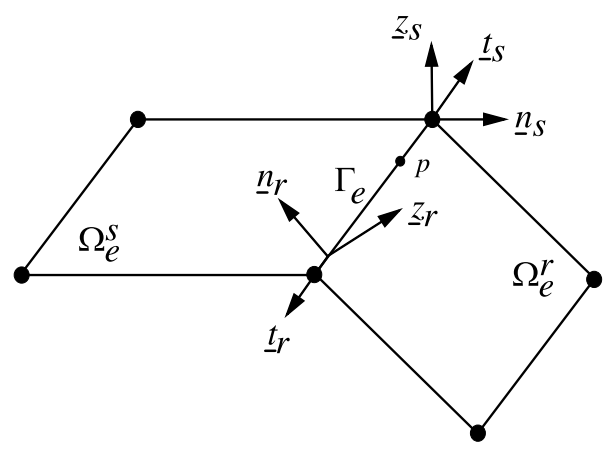

Figure 15: Interface of two non-coplanar plates

In the local basis,

$$
\begin{array}{ll}
\underline{u}^{r}=u_{n}^{r} \underline{n}_{r}+u_{t}^{r} \underline{t}_{r}+u_{z}^{r} \underline{z}_{r} & \underline{\theta}^{r}=\theta_{n}^{r} \underline{n}_{r}+\theta_{t}^{r} \underline{t}_{r} \\
\underline{u}^{s}=u_{n}^{s} \underline{n}_{s}+u_{t}^{s} \underline{\underline{t}}_{s}+u_{z}^{s} \underline{z}_{s} & \underline{\theta}^{s}=\theta_{n}^{s} \underline{n}_{s}+\theta_{t}^{s} \underline{t}_{s}
\end{array}
$$

The rotation along the normal to each plate element (drilling dof), $\underline{\theta} \cdot \underline{z}$ is not defined in local coordinates.

Considering DKT or DKQ plate finite elements, the membrane displacement on the edge is interpolated with linear shape functions, while the transverse displacement is cubic. Equating displacement nodal degrees of freedoms between the two connected elements leads to the continuity of the linear part of each displacement component.

On the other hand, since $\underline{t}_{r}=-\underline{t}_{s}$, the jump of rotation field would be

$$
\underline{\theta}^{r}-\underline{\theta}^{s}=\left(\theta_{t}^{r}+\theta_{t}^{s}\right) \underline{t}_{r}+\theta_{n}^{r} \underline{n}_{r}-\theta_{n}^{s} \underline{n}_{s}
$$

For the general case of a $3 \mathrm{D}$ plate assembling, the vectors $n_{r}, t_{r}$ and $n_{s}$ are independent, therefore a consistent continuity condition is to nullify the component on the $\underline{t}_{r}$ direction only. In other words, for non co-planar plates, since the rotation normal to the plate is not defined, the rotation continuity at the plate junction is restricted to the component corresponding to the direction which is common to the two plates. Since for DKT and DKQ elements, this rotation component is linearly interpolated on the element edges, the previous condition is imposed with the nodal values of the corresponding rotation. Note that for the special case where the two plates are in the same plane, $\underline{n}_{s}=\underline{n}_{t}$ and the full continuity condition of all degrees of freedom is admissible.

\section{B. Localized compliance between subdomains: the case of a joint}

To illustrate the management of joints between subdomains within the framework of FETI-like methods, and in particular FETI-DP approach, let us consider the case of $2 \mathrm{D}$ membrane behavior between two plates for sake of simplicity, Figure 16. The same procedure is easily applicable to the case of 3D plate assembling. 


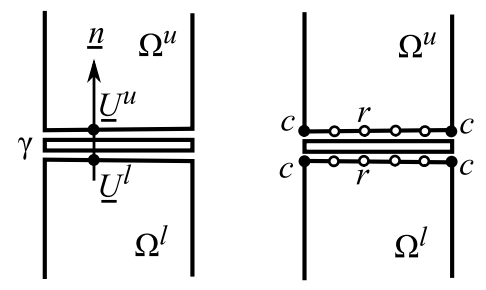

Figure 16: Elastic joint interface between two subdomains

\section{B.1. Joint model}

Consider a thin joint $\gamma$ of physical thickness $e$ between two subdomains, $\Omega^{u}$ and $\Omega^{l}$, and with a normal vector $\underline{n}$.

The assumption of linear displacement in the normal direction within the joint reads:

$$
\underline{U}=\frac{z}{e} \underline{V}+\underline{U^{l}}
$$

where $z$ is the coordinate along $\underline{n}, \underline{V}=\underline{U}^{u}-\underline{U}^{l}, \underline{U}^{u}$ (resp. $\underline{U}^{l}$ ) being the trace of displacement in subdomain $\Omega^{u}\left(\operatorname{resp} . \Omega^{l}\right)$ on the interface $\gamma$.

When the thickness $e$ is small with respect to the other dimensions, the strain within the joint is dominated by the term $\frac{1}{2 e}(\underline{V} \otimes \underline{n}+\underline{n} \otimes \underline{V})$ which is constant along the thickness. The corresponding null-strain modes are such that $\underline{U}^{u}=\underline{U}^{l}$ at each point of the interface $\gamma$ (local rigid body motions, plus extensions along $\gamma$ ).

Without initial prestress, the joint is loaded with two surfacic force densities $-\underline{F}^{u}$ and $-\underline{F}^{l}$. They should not produce any work for null-strain modes, which reads: $\underline{F}^{u}+\underline{F}^{l}=0$ (which is similar to the equilibrium of the interface $\gamma$ ). We denote in the following $\underline{\lambda}=\underline{F}^{u}=-\underline{F}^{l}$.

In the case of an elastic behavior of the joint, this load is related to the displacement jump as $\underline{\lambda}=k \underline{V}$ where $k$ is the stiffness of the joint (normal and tangential components may be different with a matrix form of the stiffness). Once a finite element discretization is used, the elastic energy of the joint is $\frac{1}{2}\left(v^{\gamma}\right)^{T} k^{\gamma} v^{\gamma}$ where $v^{\gamma}$ is the column vector of nodal values of $\underline{V}$, and $k^{\gamma}$ is the associated global stiffness matrix of the joint.

\section{B.2. FETI-DP suited formulation}

One could consider, as for the primary stiffeners, to deal with the joint as a classical subdomain, with a specific stiffness matrix due to the modeling of the joint. The problem lies in the huge null space of this stiffness matrix. Up to this point, this could be managed with a mix of the FETI and of the FETI-DP approaches to deal with it, as in $[35,36]$ but in any case, the corresponding coarse space would contain the null-energy modes of the joint and would be large in size. An adaptation of the genuine FETI-DP approach is therefore preferable to avoid such a drawback. 
Herein, we choose to consider the joint both as an elastic interface with its own constitutive relations. Therefore, once the splitting in subdomains is performed, the joint is selected as an interface and corresponding classical mappings $B^{s}$ are designed. An additional Boolean matrix $B^{\gamma}$ is added to map the additional unknown interface vector $v^{\gamma}$ to the jump on interface degrees of freedom; indeed the modified kinematic gluing constraints now reads:

$$
\sum_{s=1}^{N_{s}} B^{s} u^{s}+B^{\gamma} v^{\gamma}=0
$$

On classical perfect interfaces, $B^{\gamma}$ has null entries, and it corresponds to the classical null displacement jump; on the joint interface $\gamma$, it only names the jump as $v^{\gamma}$. The equilibrium of each subdomain $\Omega^{s}$ is unchanged, but the equilibrium of the joint must be added:

$$
k v^{\gamma}=B^{\gamma T} \lambda
$$

With the classical FETI-DP framework, the same partition of degrees of freedom (dofs) into 'corner' dofs (c-dofs) and the remaining ones ( $r$-dofs) is still used, though displacement continuity is not prescribed on elastic interface $\gamma$ since 'corner' nodes have to be duplicated on each side of the interface, Figure 16. Nevertheless, local $c$-dofs are still localized from the global $c$-dof vector $u_{c}$ as: $u_{c}^{s}=L_{c}^{s} u_{c}$ for subdomain $\Omega^{s}$, and $v_{c}^{\gamma}=L_{c}^{\gamma} u_{c}$ for joint $\gamma . L_{c}^{\gamma}$ is a signed localization matrix in order to get the coarse displacement jump $v_{c}^{\gamma}$. The kinematic constraints on $r$-dofs is:

$$
\sum_{s=1}^{N_{s}} B_{r}^{s} u_{r}^{s}+B_{r}^{\gamma} v_{r}^{\gamma}=0
$$

whose corresponding Lagrange multipliers are $\lambda$ (the gluing forces on perfect interfaces, and the normal stress vector on the elastic interface). The balance equations read:

$$
\begin{array}{r}
\forall u_{r}^{s, \star}, v_{r}^{\gamma, \star}, u_{c}^{\star} \\
\sum_{s=1}^{N_{s}}\left(u^{s, \star}\right)^{T} K^{s} u^{s}+\left(v^{\gamma, \star}\right)^{T} k^{\gamma} v^{\gamma}=\sum_{s=1}^{N_{s}}\left(u^{s, \star}\right)^{T} f^{s}+ \\
+\sum_{s=1}^{N_{s}}\left(u_{r}^{s, \star}\right)^{T} B_{r}^{s T} \lambda+\left(v^{\gamma, \star}\right)^{T} B_{r}^{\gamma T} \lambda
\end{array}
$$

Note that we do not consider herein body forces in the joint (such as a prestress) that would have lead to a generalized force vector $f^{\gamma}$. With the splitting into $c$-dofs and $r$-dofs, the local balance equations are:

- The subdomain equilibrium, identical to (6);

- The joint equilibrium:

$$
k_{r r}^{\gamma} v_{r}^{\gamma}+k_{r c}^{\gamma} L_{c}^{\gamma} u_{c}=B_{r}^{\gamma T} \lambda
$$


- The coarse problem:

$$
\begin{aligned}
\sum_{s=1}^{N_{s}} L_{c}^{s T} K_{c r}^{s} u_{r}^{s}+ & L_{c} \gamma^{T} k_{c r}^{\gamma} v_{r}^{\gamma}+ \\
& +\left(\sum_{s=1}^{N_{s}} L_{c}^{s T} K_{c c}^{s} L_{c}^{s}+L_{c}^{\gamma T} k_{c c}^{\gamma} L_{c}^{\gamma}\right) u_{c}=\sum_{s=1}^{N_{s}} L_{c}^{s T} f_{c}^{s}
\end{aligned}
$$

Condensation of (29) is not an issue since $k_{r r}^{\gamma}$ is regular due to the use of displacement jump dofs $v^{\gamma}$ to express the elastic energy. After algebraic manipulations, one gets a similar FETI-DP problem (11), but with the modified terms:

$$
\begin{aligned}
F_{I_{r r}} & =\sum_{s=1}^{N_{s}} B_{r}^{s}\left(K_{r r}^{s}\right)^{-1} B_{r}^{s T}+B_{r}^{\gamma}\left(k_{r r}^{\gamma}\right)^{-1} B_{r}^{\gamma T} \\
F_{I_{r c}} & =\sum_{s=1}^{N_{s}} B_{r}^{s}\left(K_{r r}^{s}\right)^{-1} K_{r c}^{s} L_{c}^{s}+B_{r}^{\gamma}\left(k_{r r}^{\gamma}\right)^{-1} k_{r c}^{\gamma} L_{c}^{\gamma} \\
K_{c c} & =\sum_{s=1}^{N_{s}} L_{c}^{s T} K_{c c}^{s, \star} L_{c}^{s}+L_{c}^{\gamma T} k_{c c}^{\gamma, \star} L_{c}^{\gamma} \quad \text { with } \\
k_{c c}^{\gamma, \star} & =k_{c c}^{\gamma}-k_{c r}^{\gamma}\left(k_{r r}^{\gamma}\right)^{-1} k_{r c}^{\gamma}
\end{aligned}
$$

The status of the joint is therefore half between a subdomain (with the previous contributions to the flexibility matrices), and an interface (especially

with respect to force equilibrium on the elastic interface, that lead to a single multiplier field).

Acknowledgements. The first author gratefully acknowledges the support of Iranian Ministry of Science, Research and Technology and SFERE exchange program in France for financial support during his Ph.D. thesis.

\section{References}

[1] T. Zohdi, J. T. Oden, G. J. Rodin, Hierarchical modelling of heterogeneous bodies, Computer Methods in Applied Mechanics and Engineering 138 (1996) 273-298.

[2] J. T. Oden, K. Vemaganti, N. Moës, Hierarchical modeling of heterogeneous solids, Computer Methods in Applied Mechanics and Engineering 172 (1-4) (1999) $3-25$.

[3] I. Hirai, B. P. Wang, W. D. Pilkey, An efficient zooming method for finite element analysis, International Journal for Numerical Methods in Engineering 20 (9) (1984) 1671-1683. 
[4] K. M. Mao, C. T. Sun, A refined global-local finite element analysis method, International Journal for Numerical Methods in Engineering 32 (1) (1991) $29-43$.

[5] J. D. Whitcomb, Iterative global/local finite element analysis, Computers and Structures 40 (4) (1991) 1027-1031.

[6] C. Farhat, F.-X. Roux, Implicit parallel processing in structural mechanics, in: J. T. Oden (Ed.), Computational Mechanics Advances, Vol. 2, NorthHolland, 1994.

[7] P. Le Tallec, Domain decomposition methods in computational mechanics, in: Computational Mechanics Advances, Vol. 1, North-Holland, 1994.

[8] P. Gosselet, C. Rey, Non-overlapping domain decomposition methods in structural mechanics, Archives of Computational Methods in Engineering 11-4 (2005) 1-59.

[9] C. Farhat, M. Lesoinne, K. Pierson, A scalable dual-primal domain decomposition method, Numerical Linear Algebra with Applications 7 (2000) $687-714$.

[10] C. Farhat, M. Lesoinne, P. Le Tallec, K. Pierson, D. Rixen, FETI-DP: A dual-primal unified FETI method - Part I: A faster alternative to the two-level FETI method, International Journal for Numerical Methods in Engineering 50 (7) (2001) 1523-1544.

[11] C. Farhat, F.-X. Roux, A method of finite element tearing and interconnecting and its parallel solution algorithm, International Journal for Numerical Methods in Engineering 32 (6) (1991) 1205-1227.

[12] J. Batoz, G. Dhatt, Modélisation des structures par éléments finis, Vol. 2 - Poutres et Plaques, Hermes, Paris, 1993.

[13] C. Farhat, M. Lesoinne, Mesh partitioning algorithms for the parallel solution of partial differential equations, Applied Numerical Mathematics 12 (1993) 443-457.

[14] F. Magoulès (Ed.), Mesh Partitioning Techniques and Domain Decomposition Methods, Civil-Comp Press / Saxe-Coburg Publications, 2007.

[15] A. Toselli, O. Widlund, Domain Decomposition Methods - Algorithms and Theory, Vol. Springer Series in Computational Mathematics, Springer, 2005.

[16] K. Pierson, A family of domain decomposition methods for the massively parallel solution of computational mechanics problems, Ph.D. thesis, University of Colorado at Boulder, Aerospace Engineering (2000). 
[17] J. Mandel, C. Dohrmann, Convergence of a balancing domain decomposition by constraints and energy minimization, Numerical Linear Algebra with Applications (10) (2003) 639-659.

[18] P. Vanek, J. Mandel, M. Brezina, Algebraic multigrid based on smoothed aggregation for second and fourth order problems, Computing 56 (1996) 179-196.

[19] P. Vanek, M. Brezina, J. Mandel, Convergence of algebraic multigrid based on smoothed aggregation, Numerische Mathematik 88 (2001) 559-579.

[20] P. Ladevèze, D. Dureisseix, A micro / macro approach for parallel computing of heterogeneous structures, International Journal for Computational Civil and Structural Engineering 1 (2000) 18-28.

[21] P. Ladevèze, A. Nouy, O. Loiseau, A multiscale computational approach for contact problems, Computer Methods in Applied Mechanics and Engineering 191 (2002) 4869-4891.

[22] M. Lesoinne, A FETI-DP corner selection algorithm for three-dimensional problems, in: 14th International Conference on Domain Decomposition Methods, Mexico, 2002.

[23] A. Mobasher Amini, D. Dureisseix, P. Cartraud, N. Buannic, A micromacro strategy for ship structural analysis with FETI-DP method, in: 3rd European Conference on Computational Mechanics - ECCM 2006 - Solids, Structures and Coupled Problems in Engineering, Lisbon, Portugal, 2006, pp. 233-240.

[24] D. Rixen, C. Farhat, A simple and efficient extension of a class of substructure based preconditioners to heterogeneous structural mechanics problems, International Journal for Numerical Methods in Engineering 44 (4) (1999) $489-516$.

[25] M. Bernadou, S. Fayolle, F. Léné, Numerical analysis of junctions between plates, Computer Methods in Applied Mechanics and Engineering 74 (3) (1989) 307-326.

[26] M. Bernadou, A. Cubier, Numerical analysis of junctions between thin shells. Part 1: Continuous problems, Computer Methods in Applied Mechanics and Engineering 161 (1998) 349-363.

[27] M. Bernadou, A. Cubier, Numerical analysis of junctions between thin shells. Part 2: Approximation by finite element methods, Computer Methods in Applied Mechanics and Engineering 161 (1998) 365-387.

[28] D. Dureisseix, C. Farhat, A numerically scalable domain decomposition method for the solution of frictionless contact problems, International Journal for Numerical Methods in Engineering 50 (12) (2001) 2643-2666. 
[29] P. G. Ciarlet, Theory of plates, in: Mathematical Elasticity, Vol. II of Studies in Mathematics and its Applications, North-Holland, Amsterdam, 1997.

[30] G. Geymonat, F. Krasucki, Analyse asymptotique du comportement en flexion de deux plaques collées, Comptes Rendus à l'Académie des Sciences de Paris (325) (1997) 307-314, série IIb.

[31] I. Titeux, E. Sanchez-Palencia, Junction of thin plates, European Journal of Mechanics A/Solids 19 (2000) 377-400.

[32] P. Nardinocchi, P. Podio-Guidugli, Angle plates, Journal of Elasticity 63 (2001) 19-53.

[33] P. Nardinocchi, Modelling junctions of thin plates, European Journal of Mechanics A/Solids 21 (2002) 523-534.

[34] A. M. Amini, D. Dureisseix, P. Cartraud, Multi-scale domain decomposition method for large scale structural analysis with a zooming technique: Application to plate assembly, International Journal for Numerical Methods in Engineering (2008) In press.

[35] P. Avery, G. Rebel, M. Lesoinne, C. Farhat, A numerically scalable dualprimal substructuring method for the solution of contact problems - Part I: The frictionless case, Computer Methods in Applied Mechanics and Engineering 193 (2004) 2403-2426.

[36] H. Bavestrello, P. Avery, C. Farhat, Incorporation of linear multipoint constraints in domain-decomposition-based iterative solvers - Part II: Blending FETI-DP and mortar methods and assembling floating substructures, Computer Methods in Applied Mechanics and Engineering 196 (2007) 13471368. 